\title{
The Role of Hand Therapy in Dupuytren Disease
}

Christina Turesson

The self-archived postprint version of this journal article is available at Linköping University Institutional Repository (DiVA):

http:// urn.kb.se/ resolve?urn=urn:nbn:se:liu:diva-150866

N.B.: When citing this work, cite the original publication.

Turesson, C., (2018), The Role of Hand Therapy in Dupuytren Disease, Hand Clinics, 34(3), 395-401. https:// doi.org/ 10.1016/j.hcl.2018.03.008

Original publication available at:

https:/ / doi.org/ 10.1016/j.hcl.2018.03.008

Copyright: Elsevier (12 months)

http:// www.elsevier.com/

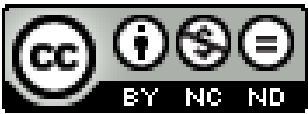


The Role of Hand Therapy in Dupuytren Disease

\section{Author name and degrees}

Christina Turesson ${ }^{\mathrm{a}, \mathrm{b}}$, ROT, PhD

\section{Author affiliations}

${ }^{a}$ Department of Hand Surgery, Plastic Surgery and Burns, and Department of Clinical and Experimental Medicine, Linköping University, Linköping, Sweden

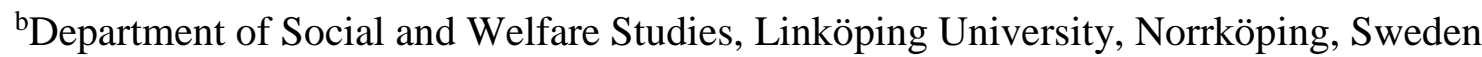

\section{Corresponding Author}

Christina Turesson

Department of Hand Surgery, Plastic Surgery and Burns

Linköping University Hospital

58185 Linkoping

Sweden

E-mail: christina.turesson@regionostergotland.se

\section{Disclosure statement}

The author reports no declarations of interest. 


\section{Key Words}

Dupuytren disease, edema, exercise, hand therapy, orthoses, patient education, scar

\section{Key Points}

- Hand therapy as preventive treatment for Dupuytren disease (DD) is uncommon with limited evidence to support its usefulness.

- Before corrective treatment, the hand therapist can perform an assessment of hand function and daily activities to assist in a systematic evaluation of outcomes after treatment.

- After corrective treatment, hand therapy is tailored to each patient's needs and consists of interventions such as orthoses, exercise, edema control, pain or scar management.

- Orthoses are usually part of the hand therapy protocol after corrective procedures for DD despite lack of strong evidence to support this intervention.

- It is recommended to provide orthoses based on the individual patient's needs instead of providing them routinely to every patient.

\section{Synopsis}

The role of hand therapy in the treatment of Dupuytren Disease (DD) varies depending on the patient and the procedure. There is limited evidence for hand therapy as a preventive treatment for DD. Before corrective treatment, the hand therapist can contribute with assessments to promote evaluation of outcome. After corrective treatment, hand therapy is tailored to each patient's needs, and consists of orthoses, exercise, edema control, and pain or scar management. Orthoses are usually part of the hand therapy protocol after corrective procedures despite lack of strong supporting evidence, and should be provided based on the individual patient's needs. 


\section{Introduction}

The overall aims of hand therapy are to prevent, restore and reverse the progression of upper limb pathologies in order to enhance the individual's ability to execute tasks and participate in daily life (1, 2). For patients with Dupuytren disease (DD), hand therapy also aims to maintain the achieved gains in finger extension and minimize the negative effects of treatment (3-5). To achieve these goals, the hand therapist commonly uses multiple treatment modalities, such as orthoses, active exercises, edema control, scar management, and patient education, depending on the patient's needs (6).

\section{Hand therapy as preventive treatment}

Patients with DD usually seek medical care when hand function is negatively affected, and this state is interfering with daily activities (7). However, it is uncommon in clinical practice that patients are referred to a hand therapist for preventive treatment, and evidence to support its use in early DD is limited. In previous research, different techniques such as orthoses, frictional massage, or heat treatment with joint stretching have been examined for the treatment of patients with palmar nodules, or limited or no finger contractures. Despite the tendency for improved digital joint extension using these techniques, alone or in combination, a systematic review has concluded there is insufficient evidence of their efficacy (8). The rationales for these treatment techniques were to promote tissue remodeling by applying low load tension through orthoses or tissue mobilization $(9,10)$, and to reduce the fibrous adhesions using cross-frictional massage (11).

The use of orthotic devices for preventive treatment for DD has been further investigated in a recently published pilot randomized controlled trial (RCT). The study investigated two orthotic devices as preventive treatment in patients with previously untreated DD or 
postoperative recurrent disease. Patients were randomized into two groups and were treated with different orthoses (tension or compression) that were to be used 20 hours a day for three months. Both groups improved from baseline average total extension deficits of 53 and 65 degrees to 32 and 46 degrees, respectively, but there were no statistically significant differences between the groups. In conclusion, the study showed that the extension deficit can be influenced with a tension or compression orthotic device used for many hours a day. However, the long-term result of using an orthotic device as a preventive or delaying strategy needs to be investigated further (12).

\section{Hand therapy before corrective treatment}

Although the finger contractures can be corrected, this does not cure DD and the disease is likely to recur or extend following treatment $(13,14)$. Preoperatively, the hand therapist can contribute an assessment of physical measures of hand function, as well as collecting patientreported outcomes to be used for short and long-term follow-up. Assessments of physical function should include measures of finger joint range of motion, grip strength, sensibility, and pain $(15,16)$. Pain has not commonly been associated with DD, but the findings of Rodrigues et al. (2014) (16) showed that 15\% of the patients with DD ( $n=110)$ experienced pain as a functional problem. Patient-reported outcomes targeting both function and activity should also be included in the preoperative assessment. In previous research, the Disability of the Arm, Shoulder and Hand (DASH) questionnaire has been the most frequently used patient-reported outcome instrument (15). Disease-specific questionnaires also exist, such as the Unité Rhumatologique des Affections de la Main (URAM) scale $(17,18)$ or the Southampton Dupuytren’s Scoring scheme (19). Questions have been raised concerning whether DASH is an appropriate instrument to use in patients with DD due to ceiling effects, or if interpretation of minimal important change is applicable to these patients (20-23). The 
URAM scale has shown acceptable responsiveness (23), but has been criticized for failing to capture common problems among patients with DD, such as difficulty with putting on a glove or putting the hand in a pocket (16). As patients with DD experience a wide range of problems in their daily lives, an individualized approach to patient-reported outcomes may be useful. For a pragmatic and patient-centered approach, patients can be asked to define tasks that are difficult to perform, and rate their performance before and after treatment (24). Furthermore, safety and social issues of hand function, i.e. the need to take special precautions due to hand function, or the avoidance of using the hand in social contexts, should also be included in patient-reported outcomes as these play an important part in patients' rating of functioning (25).

\section{Hand therapy after corrective treatment}

Hand therapy after corrective treatment consists of different modalities such as orthoses, active exercises, edema control, scar management, and patient education. The extent of intervention depends on the corrective procedure and the phases of tissue healing (4). As hand therapy is usually provided and evaluated in combination with surgery or other corrective treatment $(3,26-31)$, there is evidence to support hand therapy for patients with DD given in this combination. However, research regarding the benefits of hand therapy alone for patients with DD is lacking. Herweijer et al. (2007) (32) investigated the difference in outcomes after surgery between patients receiving hand therapy or not by comparing patients correctly referred for postoperative hand therapy compared to those incorrectly not referred for hand therapy. The hand therapy protocol used in the study consisted of static orthoses, range of motion exercises, edema reduction, and silicone dressing to limit scar hypertrophy. Orthoses were initially used for 24 hours a day, then gradually removed during day-time after the 
wound was healed, and continued at night-time for six months. No statistical significant differences were found between the groups, although the group receiving hand therapy had a tendency for greater improvement in joint mobility. The study had several limitations, including small sample size, differences between the groups regarding severity of the disease, and no randomization. These limitations make it difficult to draw definitive conclusions about the efficacy of a postoperative hand therapy protocol.

\section{Orthoses}

Orthoses have historically been used after hand surgery and trauma as a way to apply a low load prolonged stretch in order to promote soft tissue remodeling (33). For patients with DD, orthoses have commonly been used in hand therapy to maintain the achieved gains in finger extension. The orthotic intervention usually starts after reduction of dressings, and the orthosis can initially be worn 24 hours a day during the first week, and this gradually continues to night-time use in order to enable functional use of the hand. Follow-up of patients for one year after surgery for DD has shown that many patients (37\%, n=84) still use the orthosis six months after surgery (31).

Research regarding orthoses has investigated their effect on range of motion and self-reported function, scarring, and flare reactions (reactive erythema, stiffness and edema). The effect on range of motion has been investigated for both dynamic and static orthoses, and the evidence supporting this intervention is weak (34, 35). For dynamic extension orthoses, the evidence is inconclusive and of low level, showing a positive effect on severe proximal interphalangeal (PIP) joint contractures (36), but no effect on the natural course of the disease (37). Three different RCT studies have investigated the effect of static postoperative orthoses for patients with DD (38-40). All the studies showed the same result: orthoses in combination with hand therapy were no better than hand therapy alone in minimizing postoperative extension deficit of the finger joints. It is also unclear whether the use of orthoses plays a part in impaired 
finger flexion postoperatively $(31,35)$. Two studies investigated the position of the fingers in the orthosis and the effect of tension applied to the metacarpophalangeal (MCP) joints. Both studies showed that a non-tension approach, placing the MCP joints in slight flexion, was better than placing extension forces to the MCP joints during wound healing. The non-tension approach led to less scar formation and fewer flare reactions $(41,42)$.

Orthoses have also been used after treatment with needle aponeurotomy $(43,44)$ and following collagenase clostridium histolyticum (CCH) injection (45). Still, the evidence for the use of orthoses is of low level, i.e. case-study (43) or retrospective study (44), and inconclusive regarding their effect. Scherman et al. (2016) (45) compared outcomes in patients with predominantly MCP joint involvement treated with needle fasciotomy or collagenase injection at two different centers. Only one center provided patients with a night extension orthosis for three months in combination with the corrective procedure, giving the opportunity to also compare differences in outcome based on the use of orthoses. No differences were found between the groups. However, the sample size was small and no power calculation was performed for this comparison.

The need for hand therapy after CCH injection has been suggested to be limited to orthotic intervention only (46). The initial CORD 1 protocol included four months of night-time use of an orthosis (47), and many recent studies have continued with this recommendation (48-52). However, the severity of contracture of affected joints has to be taken into consideration when discussing the need for hand therapy after $\mathrm{CCH}$ injection. It has been shown that a specific post-injection orthotic intervention protocol, in combination with targeted exercises, has a positive effect on severe PIP joint contractures four weeks after treatment with injection (53). However, as the study design included no control group, further research is needed to establish evidence for the effect of post-injection orthosis beyond four weeks. 
It has been recommended to provide orthoses primarily to patients who lose finger joint extension rapidly in the early postoperative period, instead of providing it routinely to every patient (38, 39, 54). A European expert group has recommended orthotic intervention for severe cases, after open procedures, or for patients with large preoperative contractures of the PIP joint (55).

\section{Exercise}

An important part of hand therapy is to restore full finger joint range of motion. DD leads to prolonged immobilization of finger joints, which in turn leads to shortening of joint structures, e.g. joint capsules, ligaments, fascia, etc. These pre-existing changes are, together with postoperative issues, a challenge to address (56). Therefore, exercise programs are an important part of hand therapy to maximize functional use of the hand and to prevent joint stiffness (57). Range of motion exercises are usually included in postoperative hand therapy after corrective procedures for DD $(24,31,32,40,44,53,58)$. Thus, there is evidence for range of motion exercise as part of a hand therapy protocol for these patients. Michlovitz et al. (2004) concluded that there is moderate evidence for the use of exercise for managing joint contractures in the upper limbs. However, few studies have investigated finger joint contractures specifically, and the best exercise intensity or frequency is unclear (59). Therefore, to choose the most appropriate quantity of treatment or exercise for a given situation, the hand therapist needs understanding of the soft tissue response to stress. Generally, active motion provides a lower level of load than passive range of motion. Hence, active motion is started as early as possible in the inflammatory and early fibroblastic stages of tissue healing, while passive range of motion or resistance exercises can be introduced in a later stage of tissue healing $(57,60)$. For patients with DD, special attention should be given to the PIP joint as outcomes after treatment are usually better for the MCP than for the PIP joint $(51,61,62)$, and the PIP joint needs a longer time for recovery after surgical intervention 
(31). Gentle composite finger flexion exercise usually starts during the first week after surgery, and progresses during week two and three--as the wounds are healing--to include isolated tendon gliding, hook, and full fist. Aggressive exercise should be avoided to minimize complications (63).

Several factors, e.g. infection, delayed wound healing, and complex regional pain syndrome, can contribute to prolonged digital stiffness and poor hand function. In a small case series, Midgley (2010) investigated a casting motion to mobilize stiffness (CMMS) technique for four patients with DD suffering from persistent digital stiffness and a poor pattern of motion in the operated hand (56). The CMMS technique includes a non-removable plaster of Paris cast that immobilizes proximal joints in an ideal position and limits distal joint movement within a desired direction and range. This cast can be maintained for a prolonged period of time to encourage active motion at the desired joints (64). The four patients in this study were treated with a cast blocking MCP joint extension but allowing interphalangeal joint flexion in order to elongate the intrinsic muscles. The cast was worn on average for 11 weeks, and the patients performed exercises in the cast and used the casted hand in functional activities. The results showed a positive response with improved finger flexion and pattern of motion without compromising the digital extension gained during surgery (56). However, further research is needed to provide conclusive evidence for the use of the CMMS technique in patients with DD suffering from persistent joint stiffness.

\section{Other treatment modalities}

Hand therapy after corrective treatment can also involve other treatment modalities that can be applied depending on the individual patient's specific problems. Complications after surgery for DD include, among others, edema, scar hypertrophy, and flare reactions $(61,65)$. Thus, swelling, scarring, or pain may need to be addressed in hand therapy. As in all hand 
conditions treated with surgery, edema control is crucial; examples of interventions include elevation, active motion, and/or pressure garments (66). Intervention directed towards swelling, i.e. compression gloves or finger wrapping, can be needed by $30 \%$ of the patients during the first three months after fasciectomy for DD, while $12 \%$ may need intervention for scar issues (31). Scar management can consist of massage, desensitization, tape, or occlusive materials such as silicone gel sheets (63). Massage has an established role in hand therapy and is used to soften tissue and increase or maintain functional mobility $(55,67)$. Pain can be addressed with transcutaneous electrical nerve stimulation or acupuncture (31).

\section{Patient education and participation}

An important part of clinical practice is to encourage patients to be actively involved in the postoperative rehabilitation, as many of the modalities used in hand therapy rely on patients' active involvement in rehabilitation and adherence to recommendations. Patient information has also been recognized as an area that should always be addressed by the multidisciplinary team when treating patients with DD (68). As patients evaluate their health care providers' intervention, their knowledge and skills, and the interaction between them (69), tailoring information to the individual patient is important in order to reach a successful outcome (70, 71). It has been suggested that patients' compliance with the postoperative regime as a whole may influence outcomes (36), and that patients who are more engaged in their health care have better results (71-73).

\section{Summary/Discussion}

The role of hand therapy in the treatment of DD can differ significantly depending on the patient and the procedure. Thus, hand therapy can be more or less extensive due to factors such as the severity of the disease, the type of corrective procedure, the phase of tissue healing, the phase of the care process, and the patient's needs. 
Hand therapy as a preventive treatment for DD is uncommon, with limited evidence to support its usefulness. Although the extension deficit can be influenced with an orthotic device, this treatment is demanding for the patient as the orthosis needs to be worn for many hours a day, and the long-term effect on delaying finger contractures is uncertain. Before corrective treatment, the hand therapist can perform an assessment of hand function, activity limitations, and quality of life experienced by the individual, to assist in a systematic evaluation of outcomes after treatment. This can also be an opportunity to inform and prepare patients regarding the care process and rehabilitation.

The role of hand therapy is more explicit in connection with corrective treatment where different interventions can be required. The use of orthoses after corrective treatment is not self-evident. In summary, an orthotic intervention is usually part of the hand therapy protocol after corrective procedures for DD, although there is a lack of strong evidence to support this intervention. Nevertheless, it can be argued that previous research does not imply that orthoses are ineffective (27), but perhaps the use of them can be more selective. However, additional research is needed to establish indicators for which patients benefit the most from being provided with an orthosis, the optimal orthosis design and the duration of use, as well as what other interventions in a hand therapy protocol contribute to maintaining digital joint extension after surgery.

When it comes to evaluation of hand therapy, this involves challenges with multiple treatment modalities (e.g. orthoses, exercise, edema control, pain, and/or scar management) that have to be tailored to each patient's problems $(31,38)$. This individualized approach contrasts starkly with the standardization and control of intervention that is characterized in high-quality research. Hence, the efficacy of hand therapy is difficult to study. Investigation of orthotic interventions for patients with DD has been examined with several RCT studies. For other treatment modalities there is a lack of large, high-quality studies that investigate their efficacy 
specifically for patients with DD. Nevertheless, interventions directed towards range of motion, swelling, and pain or scarring are recognized as essential parts of a hand therapy protocol for patients with DD and for recovery of hand function (31). The intervention provided to patients with DD is thus based on the therapist's clinical reasoning, the patient's needs, as well as the available evidence. In the future, further evidence is needed for specific therapy interventions for patients with DD and the patients' compliance for those interventions. 


\section{References}

1. Dimick MP, Caro CM, Kasch MC, et al. 2008 practice analysis study of hand therapy. Journal of hand therapy : official journal of the American Society of Hand Therapists 2009;22(4):361-75.

2. IFSHT. IFSHT Hand Therapy Practice Profile. Secondary IFSHT Hand Therapy Practice Profile June 23, 2010 2010. https://www.ifsht.org/page/what-hand-therapy.

3. Shih B, Bayat A. Scientific understanding and clinical management of Dupuytren disease. Nat Rev Rheumatol 2010(6):715-26.

4. Hurst L. Dupuytren's Disease: surgical management. In: Skriven T, Osterman A, Fedorczyk J, Amadio P, eds. Rehabilitation of the Hand. 6 ed. Philadelphia: Elsevier Mosby, 2011:266-80.

5. Prosser R, Conolly WB. Complications following surgical treatment for Dupuytren's contracture. Journal of hand therapy : official journal of the American Society of Hand Therapists 1996;9(4):344-8.

6. Keller JL, Caro CM, Dimick MP, Landrieu K, Fullenwider L, Walsh JM. Thirty years of hand therapy: The 2014 practice analysis. Journal of hand therapy : official journal of the American Society of Hand Therapists 2016;29(3):222-34.

7. Pratt A, Byrne G. The lived experience of Dupuytren's disease of the hand. J Clin Nurs 2009;18(12):1793-802.

8. Ball C, Izadi D, Verjee LS, Chan J, Nanchahal J. Systematic review of non-surgical treatments for early dupuytren's disease. BMC musculoskeletal disorders 2016;17(1):345.

9. Larocerie-Salgado J, Davidson J. Nonoperative treatment of PIPJ flexion contractures associated with Dupuytren's disease. The Journal of hand surgery, European volume 2012;37(8):722-7.

10. Ball C, Nanchahal J. The use of splinting as a non-surgical treatment for Dupuytren's disease: a pilot study. Br Journal of Hand Therapy 2002(7):76-78.

11. Christie WS, Puhl AA, Lucaciu OC. Cross-frictional therapy and stretching for the treatment of palmar adhesions due to Dupuytren's contracture: a prospective case study. Manual therapy 2012;17(5):479-82. 
12. Brauns A, Van Nuffel M, De Smet L, Degreef I. A clinical trial of tension and compression orthoses for Dupuytren contractures. Journal of hand therapy : official journal of the American Society of Hand Therapists 2017

13. Dias JJ, Singh HP, Ullah A, Bhowal B, Thompson JR. Patterns of recontracture after surgical correction of Dupuytren disease. The Journal of hand surgery 2013;38(10):1987-93.

14. Degreef I, De Smet L. Risk factors in Dupuytren's diathesis: is recurrence after surgery predictable? Acta orthopaedica Belgica 2011;77(1):27-32.

15. Ball C, Pratt A, Nanchahal J. Optimal functional outcome measures for assessing treatment for Dupuytren's disease: a systematic review and recommendations for future practice. BMC musculoskeletal disorders 2013;14(14):131.

16. Rodrigues J, Zhang W, Scammell B, Davis T. What patients want from the treatment of Dupuytren's disease -- is the Unite Rhumatologique des Affections de la Main (URAM) scale relevant? J Hand Surg, Eur vol 2014;39(6):673-75.

17. Beaudreuil J, Lermusiaux J, Teyssedou J, et al. Multi-needle aponeurotomy for advanced Dupuytren's disease: preliminary results of safety and efficacy (MNA 1 study). Joint, bone, spine: revue du rhumatisme 2011;78(6):625-28.

18. Bernabe B, Lasbleiz S, Gerber RA, et al. URAM scale for functional assessment in Dupuytren's disease: a comparative study of its properties. Joint, bone, spine : revue du rhumatisme 2014;81(5):441-4.

19. Mohan A, Vadher J, Ismail H, Warwick D. The Southampton Dupuytren's Scoring Scheme. Journal of plastic surgery and hand surgery 2014;48(1):28-33.

20. Jerosch-Herold C, Shepstone L, Chojnowski A, Larson D. Severity of contracture and self-reported disability in patients with Dupuytren's contracture referred for surgery. Journal of hand therapy : official journal of the American Society of Hand Therapists 2011;24(1):6-10. 
21. Forget NJ, Jerosch-Herold C, Shepstone L, Higgins J. Psychometric evaluation of the Disabilities of the Arm, Shoulder and Hand (DASH) with Dupuytren's contracture: validity evidence using Rasch modeling. BMC musculoskeletal disorders 2014;15:361.

22. Rodrigues J, Zhang W, Scammell B, et al. Validity of the Disabilities of the Arm, Shoulder and Hand patient-reported outcome measure (DASH) and the Quickdash when used in Dupuytren's disease. The Journal of hand surgery, European volume 2016;41(6):589-99.

23. Rodrigues JN, Zhang W, Scammell BE, et al. Recovery, responsiveness and interpretability of patient-reported outcome measures after surgery for Dupuytren's disease. The Journal of hand surgery, European volume 2017;42(3):301-09.

24. Engstrand C, Boren L, Liedberg G. Evaluation of activity limitation and digital extension in Dupuytren's contracture three months after fasciectomy and hand therapy interventions. Journal of hand therapy : official journal of the American Society of Hand Therapists 2009;22(1):21-26.

25. Engstrand C, Krevers B, Kvist J. Factors affecting functional recovery after surgery and hand therapy in patients with Dupuytren's disease. Journal of hand therapy : official journal of the American Society of Hand Therapists 2015;28(3):255-59.

26. Au-Yong I, Wildin C, Page. A review of common practice in Dupuytren Surgery. Techniques in Hand and Upper Extremity Surgery 2005;9:178-87.

27. Sweet S, Blackmore S. Surgical and therapy update on the management of Dupuytren's disease. Journal of hand therapy : official journal of the American Society of Hand Therapists 2014;27(2):77-83.

28. Bainbridge C, Dahlin LB, Szczypa PP, Cappelleri JC, Guerin D, Gerber RA. Current trends in the surgical management of Dupuytren's disease in Europe: an analysis of patient charts. European orthopaedics and traumatology 2012;3(1):31-41. 
29. Dahlin L, Bainbridge C, Szczypa P, Cappelleri J, Guerin D, Gerber R. Current trends in the surgical management of Dupuytren's disease in Europe: the surgeon's perspective. European orthopaedics and traumatology 2012;3(1):25-30.

30. Bayat A, McGrouther DA. Management of Dupuytren's disease - clear advice for an elusive condition. Annals of the Royal College of Surgeons of England 2006;88:3-8.

31. Engstrand C, Krevers B, Nylander G, Kvist J. Hand function and quality of life before and after fasciectomy for Dupuytren disease. The Journal of hand surgery 2014;39(7):1333-43.

32. Herweijer H, Dijkstra PU, Nicolai JP, Van der Sluis CK. Postoperative hand therapy in Dupuytren's disease. Disability and rehabilitation 2007;29(22):1736-41.

33. Fess EE. A history of splinting: to understand the present, view the past. Journal of hand therapy : official journal of the American Society of Hand Therapists 2002;15(2):97-132.

34. Larson D, Jerosch-Herold C. Clinical effectiveness of post-operative splinting after surgical release of Dupuytren's contracture: a systematic review BMC musculoskeletal disorders 2008;9:104

35. Rodrigues JN, Becker GW, Ball C, et al. Surgery for Dupuytren's contracture of the fingers. The Cochrane database of systematic reviews 2015(12):CD010143.

36. Rives K, Gelberman R, Smith B, Carney K. Severe contractures of the proximal interphalangeal joint in Dupuytren's disease: results of a prospective trial of operative correction and dynamic extension splinting. Journal of hand surgery 1992;17A(6):1153-59.

37. Ebskov LB, Boeckstyns ME, Sorensen Al, Soe-Nielsen N. Results after surgery for severe Dupuytren's contracture: does a dynamic extension splint influence outcome? Scandinavian Journal of Plastic \& Reconstructive Surgery \& Hand Surgery 2000;34(2):155-60.

38. Jerosch-Herold C, Shepstone L, Chojnowski AJ, Larson D, Barrett E, Vaughan SP. Night-time splinting after fasciectomy or dermo-fasciectomy for Dupuytren's contracture: a pragmatic, multi-centre, randomised controlled trial. BMC musculoskeletal disorders 2011;12:136. 
39. Collis J, Collocott S, Hing W, Kelly E. The effect of night extension orthoses following surgical release of Dupuytren contracture: a single-center, randomized, controlled trial. The Journal of hand surgery 2013;38(7):1285-94.

40. Kemler MA, Houpt $P$, van der Horst CM. A pilot study assessing the effectiveness of postoperative splinting after limited fasciectomy for Dupuytren's disease. The Journal of hand surgery, European volume 2012;37(8):733-7.

41. Evans RB, Dell PC, Fiolkowski P. A clinical report of the effect of mechanical stress on functional results after fasciectomy for Dupuytren's contracture. Journal of hand therapy : official journal of the American Society of Hand Therapists 2002;15(4):331-39.

42. Rivlin M, Osterman M, Jacoby S, Skirven T, Ukomadu U, Lee Osterman A. The incidence of postoperative flare reaction and tissue complications in Dupuytren's disease using tensionfree immobilization. Hand 2014(9):459-65.

43. Meinel A. [Long-term static overnight extension splinting following percutaneous needle fasciotomy]. Handchirurgie, Mikrochirurgie, plastische Chirurgie : Organ der Deutschsprachigen Arbeitsgemeinschaft fur Handchirurgie : Organ der Deutschsprachigen Arbeitsgemeinschaft fur Mikrochirurgie der Peripheren Nerven und Gefasse 2011;43(5):2868.

44. Tam L, Chung Y. Needle aponeurotomy for Dupuytren contracture: effectiveness of postoperative night extension splinting. Plastic Surgery 2016;24(1):23-26.

45. Scherman P, Jenmalm P, Dahlin LB. One-year results of needle fasciotomy and collagenase injection in treatment of Dupuytren's contracture: A two-centre prospective randomized clinical trial. The Journal of Hand Surgery, Eur 2016;41E(6):577-82.

46. Naam N. Functional outcome of collagenase injections compared with fasciectomy in treatment of Dupuytren's contracture. Hand 2013(8):410-16.

47. Hurst LC, Badalamente MA, Hentz VR, et al. Injectable collagenase clostridium histolyticum for Dupuytren's contracture. The New England journal of medicine 2009;361(10):968-79. 
48. Degreef I. Collagenase Treatment in Dupuytren Contractures: A Review of the Current State Versus Future Needs. Rheumatology and therapy 2016;3(1):43-51.

49. Mickelson DT, Noland SS, Watt AJ, Kollitz KM, Vedder NB, Huang Jl. Prospective randomized controlled trial comparing 1- versus 7-day manipulation following collagenase injection for dupuytren contracture. The Journal of hand surgery 2014;39(10):1933-41

50. Coleman S, Gilpin D, Kaplan FT, et al. Efficacy and safety of concurrent collagenase clostridium histolyticum injections for multiple Dupuytren contractures. The Journal of hand surgery 2014;39(1):57-64.

51. Nydick JA, Olliff BW, Garcia MJ, Hess AV, Stone JD. A comparison of percutaneous needle fasciotomy and collagenase injection for dupuytren disease. The Journal of hand surgery 2013;38(12):2377-80.

52. Muppavarapu R, Waters M, Leibman M, Belsky M, Ruchelsman D. Clinical outcomes following collagenase injections compared to fasciectomy in the treatment of Dupuytren's contracture. Hand 2015(10):260-65.

53. Skirven TM, Bachoura A, Jacoby SM, Culp RW, Osterman AL. The effect of a therapy protocol for increasing correction of severely contracted proximal interphalangeal joints caused by dupuytren disease and treated with collagenase injection. The Journal of hand surgery 2013;38(4):684-9.

54. Samargandi OA, Alyouha S, Larouche P, Corkum JP, Kemler MA, Tang DT. Night Orthosis After Surgical Correction of Dupuytren Contractures: A Systematic Review. The Journal of hand surgery 2017;42(10):839.e1-e10.

55. Huisstede BM, Hoogvliet P, Coert JH, Friden J, European HG. Dupuytren disease: European hand surgeons, hand therapists, and physical medicine and rehabilitation physicians agree on a multidisciplinary treatment guideline: results from the HANDGUIDE study. Plastic and reconstructive surgery 2013;132(6):964-76. 
56. Midgley R. Use of casting motion to mobilize stiffness to regain digital flexion following Dupuytren's fasciectomy. Hand Therapy 2010(15):45-51.

57. Comer GC, Clark SJ, Yao J. Hand Therapy Modalities for Proximal Interphalangeal Joint Stiffness. The Journal of hand surgery 2015;40(11):2293-6.

58. Glassey N. A study of the effect of night extension splintage on post-fasciectomy Dupuytren's patients. British Journal of Hand Therapy 2001;6(3):89-94.

59. Michlovitz S, Harris BA, Watkins MP. Therapy Interventions for improving joint range of motion: a systematic review. Journal of Hand Therapy 2004;17:118-31.

60. Glasgow C, Tooth LR, Fleming J. Mobilizing the stiff hand: combining theory and evidence to improve clinical outcomes. Journal of hand therapy : official journal of the American Society of Hand Therapists 2010;23(4):392-400.

61. Crean S, Gerber R, Hellio Le Graverand M, Boyd D, Cappelleri J. The efficacy and safety of fasciectomy and fasciotomy for Dupuytren's contracture in European patients: a structured review of published studies. J Hand Surg Eur 2011;36(5):396-407.

62. Badalamente MA, Hurst LC, Benhaim P, Cohen BM. Efficacy and safety of collagenase clostridium histolyticum in the treatment of proximal interphalangeal joints in dupuytren contracture: combined analysis of 4 phase 3 clinical trials. The Journal of hand surgery 2015;40(5):975-83.

63. Evans RB. Therapeutic management of Dupuytren's contracture. In: Skriven T, Osterman A, Fedorczuk J, Amadio P, eds. Rehabilitation of the hand and upper extremity. 6 ed. Philadelphia: Elsevier Mosby, 2011:281-88.

64. Colditz JC. Plaster of Paris: the forgotten hand splinting material. Journal of hand therapy : official journal of the American Society of Hand Therapists 2002;15(2):144-57.

65. Denkler K. Surgical complications associated with fasciectomy for dupuytren's disease: a 20-year review of the English literature. Eplasty 2010;10:e15. 
66. Villeco JP. Edema: Therapist's management. In: Skirven T, Lee Osterman A, Fedorczyk J, Amadio P, eds. Rehabilitation of the hand and upper extremity. 6 ed. Philadelphia: Elsevier Mosby, 2011:845-57.

67. Shin TM, Bordeaux JS. The role of massage in scar management: a literature review. Dermatologic surgery : official publication for American Society for Dermatologic Surgery [et al.] 2012;38(3):414-23.

68. Jones L. Scar management in hand therapy - is our practice evidence based? British journal of Hand Therapy 2005;10(2):40-46.

69. Pomey MP, Ghadiri DP, Karazivan P, Fernandez N, Clavel N. Patients as partners: a qualitative study of patients' engagement in their health care. PloS one 2015;10(4):e0122499.

70. Moorhead J, Cooper C, Moorhead P. Personality type and patient education in hand therapy. Journal of hand therapy : official journal of the American Society of Hand Therapists 2011;24(2):147-53.

71. Engstrand C, Kvist J, Krevers B. Patients' perspective on surgical intervention for Dupuytren's disease - experiences, expectations and appraisal of results. Disability and rehabilitation 2016:1-12.

72. Gruber JS, Hageman M, Neuhaus V, Mudgal CS, Jupiter JB, Ring D. Patient activation and disability in upper extremity illness. The Journal of hand surgery 2014;39(7):1378-83.

73. Hibbard JH, Greene J. What the evidence shows about patient activation: better health outcomes and care experiences; fewer data on costs. Health affairs 2013;32(2):207-14. 